\title{
A FORMAÇÃO DE CONSTELAÇÕES: 0 CASO DA INDÚSTRIA GLOBAL DE TRANSPORTE AÉREO
}

RESUMO

Constelações são alianças constituídas por empresas autônomas que competem entre si por clientes e membros no mesmo setor ou em setores similares. Empresas de transporte aéreo não somente vêm formando agrupamentos formais multilaterais, que aqui foram denominados de constelações explícitas, como também têm se engajado em uma rede de laços bilaterais que constituem grupos alternativos de empresas com maiores laços umas com as outras do que com empresas de fora do grupo, o que denominamos de "constelações implícitas". Este estudo mostra que constelações explícitas tendem a crescer atraindo empresas que possuem recursos diversos e que estejam associadas a membros-chave por meio de um grupo implícito. Ao mesmo tempo, membros de um grupo explícito tendem a criar associações implícitas com parceiros que possuam recursos similares aos seus, criando assim um grupo de empresas adjacente à constelação explícita. Outra descoberta deste estudo é a de que as associações explícitas tendem a apresentar uma inércia maior ao longo do tempo do que as associações implícitas, possivelmente devido aos grandes investimentos e aos compromissos contratuais necessários para a formação de grupos explícitos.

\section{Sérgio G. Lazzarini \\ Faculdades Ibmec}

\section{Thiago A. Z. Joaquim}

Faculdades Ibmec

\begin{abstract}
Constellations are alliances among multiple autonomous firms, such that these groups compete against each other in the same or similar industries for both clients and members. Airline carriers, in particular, have not only formed formal groupings in a multilateral fashion which we call "explicit constellations" - but have also engaged in a web of bilateral ties configuring alternative groups of firms that have more bilateral ties to one another than to firms outside their group - which we call "implicit constellations". We find that explicit constellations tend to grow by luring firms holding diverse resources and that are associated with key members through an implicit group. At the same time, members of an explicit group tend to create implicit associations with partners holding resources that are similar to their own resources, thereby creating a cluster of firms adjacent to the explicit constellation. Explicit associations also tend to exhibit more inertia over time than implicit ones, possibly due to the larger non-redeployable investments and contractual commitments required for the formation of explicit groups.
\end{abstract}

PALAVRAS-CHAVE Constelações, alianças estratégicas, redes, estratégia cooperativa, setor de transporte aéreo. KEY WORDS Constellations, strategic alliances, networks, cooperative strategy, airline industry. 


\section{INTRODUÇÃo}

Constelações são alianças entre diversas empresas autônomas que competem umas contra as outras por clientes e membros no mesmo setor ou em setores similares (Gomes-Casseres, 1994). Evidências sobre a formação desses grupos permeiam a literatura, incluindo indústrias diversas, como a de computadores e microprocessadores (Axelrod et al., 1995; Hwang e Burgers, 1997; Vanhaverbeke e Noorderhaven, 2001); de telecomunicações (Joshi et al., 1998); de serviços financeiros (Domowitz, 1995); a automobilística (Burgers et al., 1993; Garcia-Pont e Nohria, 2002; Nohria e Garcia-Pont, 1991); e de aviação global (Hanlon, 1999; Ter Kuile, 1997).

A formação de alianças entre empresas internacionais de transporte aéreo, por exemplo, vem permitindo certas facilidades aos viajantes, tais como a possibilidade de escolher dentre as diversas rotas substitutas oferecidas por grupos de empresas que exploram rotas complementares. O surgimento dessas associações compostas por diversas empresas levou alguns acadêmicos a proporem que o locus de competição vem se deslocando das empresas individuais para grupos de empresas que colaboram umas com as outras (Gomes-Casseres, 1996; Gulati, 1998). Assim, decisões para formação de constelações provavelmente possuem profundas implicações estratégicas, já que o desempenho da empresa pode depender de maneira vital do grupo com o qual a empresa decide se unir.

Apesar da crescente formação de constelações em diversos setores, sabemos relativamente pouco sobre como esses grupos surgem, já que pesquisas anteriores sobre o tema focaram apenas a formação de laços bilaterais entre empresas (Chung e Singh, 2000; Gulati, 1995b; Gulati e Gargiulo, 1999; Li e Rowley, 2002; Martin e Park, 2002; Stuart, 1998). Ainda que alguns estudos tenham de fato analisado constelações (Burgers et al., 1993; Nohria e Garcia-Pont, 1991; Vanhaverbeke e Noorderhaven, 2001; Walker, 1988), eles não examinam em detalhes os determinantes da formação desses grupos e a dinâmica envolvida no processo. Em vez disso, esses estudos tiveram como objetivo analisar a composição das constelações de maneira estática, observando quais empresas estavam associadas com quais outras empresas em um determinado período.

O objetivo do presente estudo é preencher a lacuna deixada pelas pesquisas sobre o tema. Em específico, busca-se analisar o modo como surgem e evoluem as constelações entre empresas. O setor escolhido para análise é o de empresas de transporte aéreo, visto que ele vem testemunhando um aumento progressivo no número de alianças. Tal aumento pode ser em parte explicado pelo fato de que os obstáculos regulatórios do setor impedem o acesso a recursos e mercados globais por meio de aquisição de instalações de aeroportos ou de empresas domésticas de transporte aéreo (Hanlon, 1999; Oum e Yu, 1998). Assim, as alianças tornaram-se um mecanismo crucial para que as empresas de transporte aéreo absorvessem as externalidades entre empresas na forma de fluxos de tráfego internacional (Oum e Yu, 1998; Park e Zhang, 2000; Park e Martin, 2001).

Os acordos multilaterais no setor aéreo datam de meados dos anos 1990, quando envolviam acordos de cooperação de marketing, relacionados a programas de vôo e promoções - incluindo investimentos na marca comum -, além de acesso conjunto às instalações controladas por membros individuais. Estimativas indicam que esses agrupamentos contribuíram com quase $60 \%$ do tráfego global em 2001, representando US\$ 203,3 bilhões em receitas (Baker, 2001). Dado o pioneirismo e a relevância estratégica da formação de alianças no setor aéreo, ele torna-se um rico cenário para uma investigação detalhada sobre a origem e evolução das constelações.

A seguir, apresentamos uma definição dos conceitos empregados neste estudo, particularmente do conceito de constelações explícitas e implícitas. Seguem as hipóteses e a metodologia do estudo. Por fim, são apresentados os resultados e as principais conclusões da pesquisa, com recomendações para futuras investigações sobre o tema.

\section{CONSTELAÇÕES EXPLÍCITAS E IMPLÍCITAS}

Estamos interessados em estudar a co-evolução de dois tipos de constelações: as explícitas e as implícitas (Lazzarini, 2003). Constelações explícitas envolvem acordos formais, publicamente conhecidos e com um caráter multilateral, na medida em que tendem a ser amplos e gerais; ou seja, aplicáveis a todos os membros. Na maioria das vezes, essas constelações estão associadas a alguma marca e a comitês especificamente encarregados de administrar os interesses do grupo. No setor de aviação, constelações explícitas constituem grupos multilaterais, dos quais os mais notáveis são a Star Alliance (United Airlines, Lufthansa, SAS), Oneworld (American Airlines, British Airways, 
Quantas) e Sky Team (Delta Airlines, Air France). As coalizões de bolsas financeiras internacionais (Domowitz, 1995) e os consórcios formais de Pesquisa \& Desenvolvimento (Axelrod et al., 1995; Hwang e Burgers, 1997; Vanhaverbeke e Noorderhaven, 2001) também são exemplos de constelações explícitas.

Constelações implícitas, por outro lado, são grupos informais com base em uma estrutura bilateral de acordos entre empresas. Nesse tipo de estrutura, os membros mantêm relativamente mais ligações bilaterais uns com os outros do que com empresas de fora da constelação (Nohria e Garcia-Pont, 1991). Assim, enquanto as constelações explícitas podem ser descritas como alianças amplas envolvendo diversas empresas (Das e Teng, 2002), as constelações implícitas representam coleções de diversas alianças bilaterais. Em alguns casos, as constelações implícitas também podem ser "versões" expandidas de constelações explícitas, na medida em que podem incluir empresas direta ou indiretamente ligadas a membros-chave - não necessariamente todos - destas últimas.

No setor de aviação, há evidências de que as constelações implícitas existiam antes mesmo do surgimento de grupos explícitos. Consistiam, na maioria dos casos, de coalizões expandidas que tinham, como grupo central, constelações explícitas. A Figura 1 ilustra a existência, no início de 2000, de um subgrupo de empresas direta ou indiretamente associado à constelação - explícita - formada pela Star Alliance. As linhas finas da figura representam laços bilaterais - alianças ou laços de propriedade entre duas empresas -; o círculo pontilhado inclui os membros da Star Alliance naquele período. Algumas empresas que não eram membros formais do grupo explícito estavam de algum modo ligadas a alguns dos membros formais do grupo.

Por exemplo, a British Midland Airways (bmi) possuía laços bilaterais com Air Canada, Air New Zealand, Austrian Airlines, Lufthansa, SAS e United Airlines, mas não era membro da Star Alliance até julho de 2000, quando então se juntou à constelação explícita. Outras empresas de transporte aéreo, como Emirates, Malaysia Airlines, South African Airways e Virgin Atlantic, não eram membros formais do grupo, embora mantivessem com ele laços bilaterais. Esse grupo expandido, que incluía tanto os membros da constelação explícita quanto os não-membros, ligados bilateralmente às empresas do grupo, sugeria a existência de uma constelação implícita anterior à constelação explícita.

Figura 1 - Exemplo de constelação explícita e implícita - Star Alliance, ano 2000.

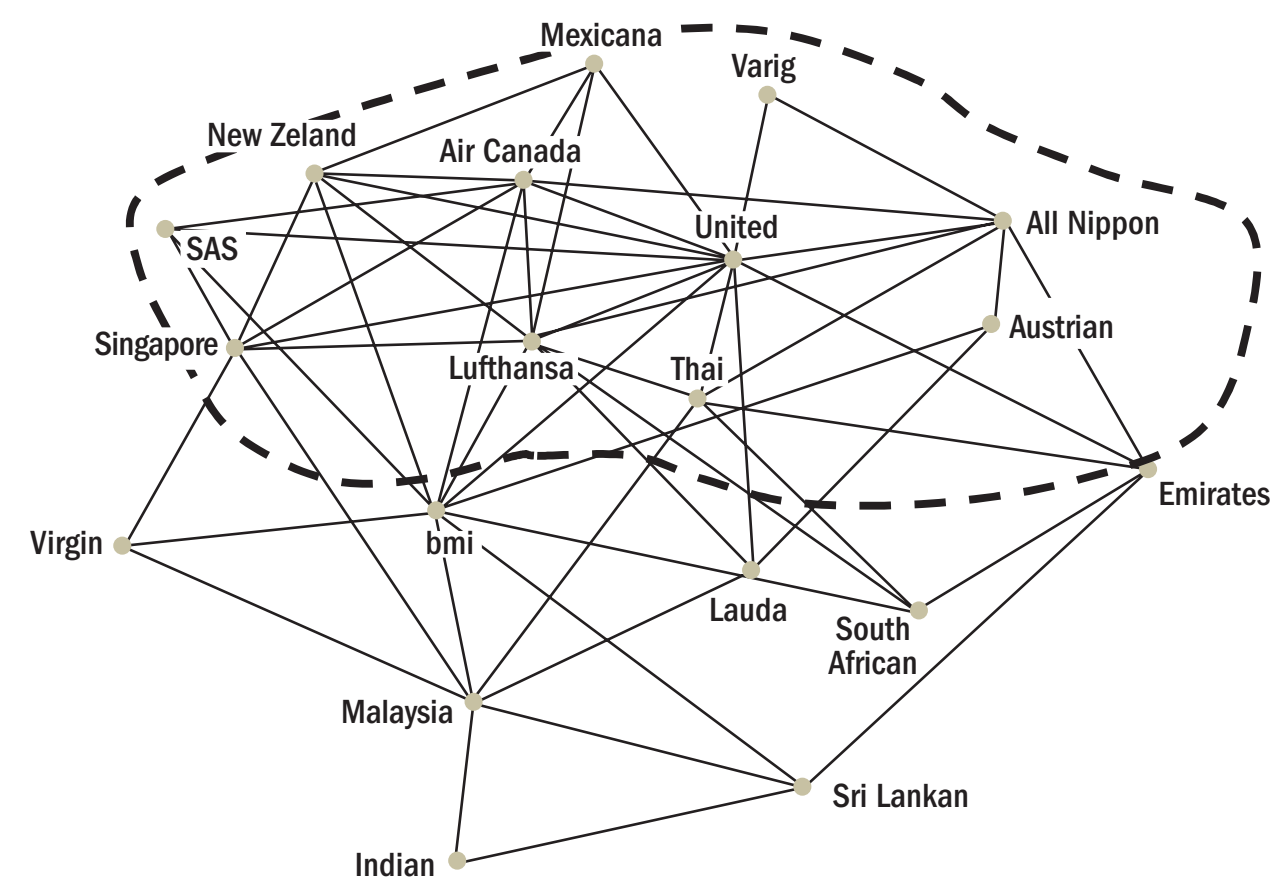


O caso da bmi levanta uma série de questões relacionadas à formação e co-evolução de constelações explícitas e implícitas. Por exemplo, poderíamos fazer uma generalização a partir dele e afirmar que a expansão de uma constelação explícita se baseia na inclusão de empresas previamente associadas aos membros do grupo formal por meio de constelações implícitas? Em outras palavras, as associações implícitas entre empresas tendem a se tornar mais "formalizadas" com o tempo? Ou, alternativamente, será que a formação de uma configuração explícita induz a uma reorganização dos laços bilaterais entre as empresas, de forma que a configuração de constelações implícitas se transforme ao longo do tempo? Essas são algumas das perguntas que nortearam este estudo. Vejamos como foram operacionalizadas na forma de hipóteses.

\section{HIPÓTESES}

Um dado comum na literatura sobre alianças é o de que as empresas selecionam, como futuros parceiros, aqueles com os quais transacionaram no passado (Gulati, 1995b; Gulati e Gargiulo, 1999; Li e Rowley, 2002; Martin e Park, 2002; Stuart, 1998). Por meio de interação contínua, as empresas desenvolvem normas comuns e confiança umas nas outras - ou, de maneira mais generalizada, "capital social" -, estimulando assim a continuidade do relacionamento (Gulati, 1995a; Macneil, 1980; Ring e Van de Ven, 1994). As empresas também tendem a persistir em um dado curso de ação e, dessa forma, manter sua associação por meio do desenvolvimento de rotinas conjuntas e investimentos comuns irrecuperáveis (Blau, 1964; Levinthal e Fichman, 1988; Levinthal e March, 1993).

Estamos particularmente interessados em conhecer as possíveis diferenças entre constelações implícitas e explícitas no que se refere ao estabelecimento de parcerias duradouras. Conforme mencionado, constelações explícitas envolvem acordos multilaterais que, conjuntamente, formalizam a associação de toda a rede de empresas e, em alguns casos, envolvem investimentos comuns. Já as constelações implícitas são agrupamentos informais cuja composição é basicamente definida pela configuração dos laços bilaterais entre as empresas envolvidas; diferentemente das constelações explícitas, elas não envolvem formalizações ou investimentos comuns. Assim, apesar de as constelações implícitas poderem exibir um tipo de inércia devido aos custos para reorganização dos laços bilaterais, tal inércia tenderá a ser ainda maior nos grupos explícitos devido aos complexos compromissos contratuais e aos investimentos conjuntos, que aumentam substancialmente os custos no caso de um rompimento da parceria. Disso decorre nossa primeira hipótese, abaixo apresentada.

Hipótese 1: A tendência de as empresas permanecerem na mesma constelação ao longo do tempo é mais forte no caso das constelações explícitas do que das implícitas.

Estamos também interessados na identificação da co-evolução das constelações implícitas e explícitas. A participação em uma mesma constelação implícita deve resultar em participação na mesma constelação explícita. Observamos anteriormente que constelações implícitas representam, em alguns casos, versões expandidas de constelações explícitas. Assim, é muito provável que a expansão de um grupo explícito, por meio da atração de membros de constelações implícitas correspondentes - como o caso da bmi discutido anteriormente -, gere uma economia nos custos de busca de um novo parceiro e permita que uma aliança mais ampla seja construída a partir dos laços bilaterais existentes.

Além disso, constelações implícitas podem ser precursoras de grupos explícitos; ou seja, elas podem se formalizar ao longo do tempo. Tal formalização possui diversas vantagens. Primeiro, a criação de procedimentos padronizados de troca em grupos explícitos tende a aumentar a compatibilidade de produção e sistemas de marketing entre os membros do grupo, elevando a capacidade de absorção de externalidades tecnológicas ou mercadológicas (Schilling e Steensma, 2001; Thompson, 1967). Segundo, a existência de uma diretoria e de comitês formais nas constelações explícitas facilita o controle e a melhoria do processo de tomada de decisão coletiva (Farrell e Saloner, 1988). E, finalmente, a formalização de associações entre empresas e a criação de marcas trazem legitimidade e visibilidade ao grupo, ajudando na atração de novos clientes e agentes de suporte, como fornecedores e investidores (Human e Provan, 2000; Katz e Shapiro, 1994). Disto segue a primeira parte de nossa segunda hipótese.

Hipótese 2a: Empresas que já pertenceram a uma mesma constelação implícita no passado provavelmente pertencerão à mesma constelação explícita no futuro. 
Podemos também supor que a participação em uma constelação explícita implicará a participação comum em uma constelação implícita no futuro. Alguns grupos explícitos criam acordos de exclusividade com o objetivo de reduzir as externalidades que um membro pode gerar, via laços bilaterais, para empresas que não pertencem ao grupo. Da mesma forma, a presença de relações externas extensivas provavelmente reduzirá o comprometimento das empresas com o grupo, desestabilizando-o (Jones et al., 1998). Além disso, visto que as constelações explícitas aumentam as ações conjuntas das empresas por meio de comitês e outros mecanismos de governança, o contato entre as empresas tenderá a ser muito mais intenso (McPherson, 1982) e, por conseqüência, poderá gerar novos acordos bilaterais, além das atividades do grupo explícito. Como conseqüência, podemos observar que os acordos bilaterais entre empresas que compõem uma constelação implícita podem vir a complementar os acordos feitos por essas mesmas empresas em suas constelações explícitas. Isso sugere que, ao longo do tempo, as empresas provavelmente reorganizarão suas associações bilaterais em um padrão consistente com seus laços coletivos no grupo explícito. Disto segue a segunda parte de nossa segunda hipótese.

Hipótese 2b: Empresas que pertenceram às mesmas constelações explícitas no passado provavelmente pertencerão à mesma constelação implícita no futuro.

Nossa última hipótese relaciona-se com o modo como o perfil de recursos das empresas poderá influenciar no surgimento de constelações explícitas e implícitas. A possibilidade de absorver externalidades entre empresas aumenta à medida que os membros mantêm recursos complementares - em outras palavras, quando o uso de um determinado recurso aumenta na medida em que ele é utilizado conjuntamente com outros recursos oferecidos pelos parceiros. A tendência é que isso ocorra quando as empresas desempenham papéis especializados e quando contribuem para a constelação com diversos recursos que podem ser combinados entre si (Grandori e Soda, 1995; Richardson, 1972; Teece, 1992).

Por exemplo, uma empresa de transporte aéreo pode absorver o tráfego de outras empresas oferecendo rotas alternativas. Uma vez que as regulamentações internacionais impedem uma empresa de transporte aéreo de possuir infra-estrutura estrangeira, essa em- presa pode complementar sua rede de rotas fazendo parcerias com empresas que controlam hubs diferentes, reunindo assim tráfego advindo de diferentes regiões. Esse fato pode sugerir que as constelações serão formadas por empresas que possuam recursos diversos e complementares (Gomes-Casseres, 1994), embora também seja possível argumentar que a proximidade e similaridade de recursos podem facilitar o monitoramento entre empresas, o compartilhamento de informações e a busca de metas comuns (Caves e Porter, 1977; Darr e Kurtzberg, 2000; Gnyawali e Madhavan, 2001; Kraatz, 1998).

Defendemos neste estudo que o efeito líquido da diversidade de recursos irá depender do fato de a constelação ser explícita ou implícita. É provável que o aumento da diversidade de recursos em constelações explícitas não sobrecarregue a habilidade do grupo de coordenar suas atividades, pois os mecanismos de coordenação formais existentes nessas constelações irão atenuar as dificuldades envolvidas na articulação de recursos diferentes. Uma vez que as constelações implícitas não possuem tais mecanismos formais, a diversidade de recursos poderá minar o nível de coordenação entre as empresas, e isso de forma mais intensa do que nos grupos explícitos. Disto decorre nossa última hipótese.

Hipótese 3: A diversidade de recursos terá uma influência maior sobre a formação de uma constelação explícita do que sobre a formação de uma constelação implícita.

\section{METODOLOGIA}

\section{Dados}

A amostra foi formada por 75 empresas globais de aviação que, em 2000, foram responsáveis por aproximadamente $81 \%$ do total de tráfego de passageiros distribuídos em 54 países. A observação dessas empresas foi realizada de 1995 a 2000. Os dados foram obtidos, em sua maioria, do World Air Transport Statistics e compilados pela International Air Transport Association (IATA), que fornece informações sobre operações das empresas de aviação, e da revista Airline Business, que apresenta pesquisas anuais sobre alianças bilaterais, laços de propriedade entre as empresas de transporte aéreo, assim como a composição de constelações explícitas nesse setor. O critério para incluir uma empresa de transporte aéreo como um membro de uma constelação explícita foi a existência de um 
anúncio, feito por essa empresa, sobre sua associação a um grupo; tal anúncio deveria ter sido feito entre janeiro e junho de cada ano analisado. No caso de dissolução, foi adotado como critério que o grupo continuaria sendo considerado apenas no caso de o anúncio haver sido feito na segunda metade do ano em questão.

Diferentemente das constelações explícitas, cuja identificação é facilmente obtida por meio de fontes públicas, a composição de grupos implícitos é mais difícil de ser determinada; para tanto, são exigidos métodos específicos. Tomando como base trabalhos anteriores (Nohria e Garcia-Pont, 1991; Vanhaverbeke e Noorderhaven, 2001; Walker, 1988), foi adotada neste estudo a análise de cluster para demarcar as fronteiras de constelações implícitas, tomando como base a matriz de laços bilaterais entre as empresas de transporte aéreo incluídas na amostra.

Para a criação da matriz de laços bilaterais, tomouse como critério que a existência de tais laços entre duas empresas ocorre quando elas possuem uma aliança bilateral ou uma relação de propriedade, que ocorre quando pelo menos uma das empresas possui uma parcela do patrimônio da outra. Do contrário, admitiu-se que não havia laço. Essa matriz foi construída em função dos anos analisados. O algoritmo de cluster aqui adotado baseou-se na otimização conhecida como tabu search (Glover, 1989), disponível no software Ucinet 5.0 (Borgatti et al., 1999). A função do algoritmo é maximizar uma função "ajuste" baseada na "proximidade" média entre os membros de um grupo, definidos em termos de existência de laços bilaterais recíprocos, dado um número pré-especificado de grupos ou divisões. Assim, o algoritmo possui uma regra clara para otimizar a composição dos grupos (Lawless e Anderson, 1996). Em alguns casos, o algoritmo agrupa empresas de transporte aéreo que não possuem laços diretos ou que exibem apenas laços em pares e isolados. Essas empresas provavelmente possuem um padrão de laços bilaterais que não permite que sejam classificadas em nenhum grupo. Assim, considerou-se que tais tipos de empresas não estão vinculados a nenhum grupo implícito.

Uma decisão crítica em análise de cluster é a definição do número ideal de divisões, que, no caso deste estudo, correspondeu ao número de constelações implícitas a ser encontrado. Foram escolhidas cinco divisões, porque no último ano considerado na amostra, 2000, havia cinco constelações explícitas. Uma vez que as constelações implícitas podem ser precursoras de grupos explícitos ou versões expandidas desses gru- pos, é natural encontrar um padrão de agrupamento que possua alguma correspondência com a configuração de constelações explícitas.

Em vista do fato de que as rotas transatlânticas entre Europa e Estados Unidos são consideradas alvoschave para a formação de alianças globais em aviação, foi assumido neste estudo que as maiores empresas de transporte aéreo dos Estados Unidos serão participantes centrais em cada grupo aqui considerado. Na amostra selecionada, quatro empresas de transporte aéreo dos Estados Unidos podem ser consideradas participantes-chave internacionais, a saber: American Airlines, Delta Airlines, Northwest Airlines e United Airlines, sugerindo, assim, quatro constelações. Por fim, o quinto agrupamento foi obtido pela adição de um grupo de empresas de transporte aéreo antigamente liderado pela Swissair. A composição dos grupos explícitos e implícitos analisados neste estudo é mostrada nas Tabelas 1 e 2, respectivamente.

\section{Variável dependente}

Um caminho natural para modelar a decisão de uma empresa em se juntar a uma constelação particular seria utilizar um modelo de múltipla escolha para descrever qual grupo uma determinada empresa escolhe. Nesse caso, seria possível a criação de um conjunto de variáveis dependentes que codificasse a participação das empresas em cada grupo. No entanto, essa abordagem é problemática, uma vez que o conjunto de escolhas disponíveis para as empresas não é fixo, invalidando dessa forma a pressuposição de modelos usuais de múltipla escolha em que as categorias de escolha são exógenas (Maddala, 1983). Na verdade, constelações são continuamente formadas, da mesma forma que as empresas, endogenamente, escolhem se aliar e formar novos grupos.

Assim, em vez de descrever qual o grupo escolhido pelas empresas, a opção deste estudo foi pela modelagem de quais empresas decidiram se associar umas com as outras na mesma constelação. No entanto, essa abordagem pode se tornar ineficiente se considerarmos todas as coalizões que as empresas podem vir a formar. Assim, $n$ empresas podem criar associações envolvendo dois, três, quatro ou mais agentes, incluindo a grande coalizão com $n$ membros. Para simplificar a análise, avaliou-se a participação conjunta em constelações a partir de pares de empresas. Especificamente, foi descrito se duas empresas de transporte aéreo da amostra foram observadas na mesma constelação explícita ou implícita em um dado ano. 
Convém observar que empresas de transporte aéreo podem estar associadas de três maneiras diferentes: pertencendo à mesma constelação implícita; pertencendo à mesma constelação explícita; ou estando bilateralmente vinculadas umas às outras. Essas alternativas não são mutuamente excludentes. Como referência, observemos a Figura 1. A bmi não era membro de nenhum grupo explícito no início de 2000; porém, ela era parte da mesma constelação implícita que incluía diversos membros da Star Alliance - conforme indicado pela análise de cluster. Da mesma forma, a Virgin e a South African Airways eram membros da mesma constelação implícita, mas não estavam bilateralmente vinculadas; em vez disso, estavam indiretamente associadas por meio da bmi. Finalmente, embora a Varig e a Mexicana tenham sido membros do mesmo grupo explícito - Star Alliance -, não foi observado nenhum laço bilateral entre as duas.

Foram então criadas duas variáveis dependentes para monitorar a adesão das empresas de transporte

Tabela 1 - Descrição das constelações implícitas.

\begin{tabular}{|c|c|c|c|c|c|c|c|}
\hline \multirow{2}{*}{ ANO } & \multirow{2}{*}{ CÓDIGO } & \multirow{2}{*}{ MEMBROS } & \multicolumn{5}{|c|}{ DENSIDADE $^{\mathrm{a}}$} \\
\hline & & & 1 & 2 & 3 & 4 & 5 \\
\hline \multirow{5}{*}{1995} & 1 & LIN, AMX, ALA, AW, BA, CO, GB, KLM, MEX, NW, USA. & .22 & & & & \\
\hline & 2 & AFL, AC, CHI, AFR, ANA, AUS, BAL, CSA, DL, FIN, IBR, LOT, LFH, MLV, SAB, SWR, TAR, THY. & .04 & .36 & & & \\
\hline & 3 & ARG, IND, ANS, BMI, EMI, MA, RA, SLA, TAP, TWA, UA, VIR. & .00 & .06 & .24 & & \\
\hline & 4 & ALG, ALI, CYP, EGY, GUL, IRA, KOR, OLY, RAM, SAU, SYR, TUN. & .03 & .06 & .04 & .23 & \\
\hline & 5 & ANZ, AA, CAI, CP, JAS, JA, LAB, QUA, SAS, SIN, SAA, TAI, VRG, VSP. & .04 & .06 & .04 & .02 & .23 \\
\hline \multirow{5}{*}{1996} & 1 & LIN, LIB, ANZ, AW, BA, CAI, GB, LCH, NW, QUA, SAS, USA, VRG. & .22 & & & & \\
\hline & 2 & AFL, AMX, ALG, AFR, BAL, CRO, EGY, MEX, RAM, TUN, THY. & .00 & .24 & & & \\
\hline & 3 & ARG, AA, ANS, BMI, CP, JAS, JA, MA, RAJ, SIN, SAA, SLA, TAP, TAI, VIR. & .05 & .01 & .25 & & \\
\hline & 4 & AC, CHI, ALI, ANA, AUS, CO, CSA, DL, FIN, IBR, KOR, LAU, LOT, LFH, MLV, SAB, SWR, TAR. & .05 & .06 & .08 & .35 & \\
\hline & 5 & IND, CYP, EMI, GUL, IRA, KLM, LAB, OLY, SAU, SYR, TWA, UA, VSP. & .03 & .03 & .04 & .04 & .22 \\
\hline \multirow{5}{*}{1997} & 1 & LIN, CHI, ALA, AW, DL, EL, FIN, KLM, KOR, NW, SAB, SIN, TAP. & .26 & & & & \\
\hline & 2 & AFL, AMX, AFR, ALI, AUS, BAL, CO, CRO, CSA, IBR, LOT, MLV, SWR, TAR, THY, UKR. & .08 & .39 & & & \\
\hline & 3 & ARG, AC, IND, ANZ, ANS, BMI, CP, EMI, LAU, LFH, MA, SAS, SAA, SLA, TAI, UA, VIR. & .04 & .05 & .29 & & \\
\hline & 4 & ALG, CYP, EGY, GUL, IRA, LAB, OLY, RAM, SAU, SYR, TUN, VSP. & .01 & .06 & .03 & .20 & \\
\hline & 5 & LIB, ANA, AA, BA, CAI, GB, JAS, JA, MEX, QUA, VRG. & .04 & .04 & .07 & .01 & .25 \\
\hline \multirow{5}{*}{1998} & 1 & LIN, AOM, CRO, EGY, LAU, MA, OLY, RAJ, SAB, SLA, TAP,THY. & .20 & & & & \\
\hline & 2 & AFL, AMX, CHI, AFR, IND, ALI, AUS, BAL, CSA, DL, FIN, IBR, KOR, LOT, MLV, SWR, TAR, UKR. & .10 & .39 & & & \\
\hline & 3 & ARG, AW, AA, BA, CAI, CO, JAS, JA, LCH, LAB, QUA, VSP. & .01 & .07 & .26 & & \\
\hline & 4 & ALG, ALA, CYP, GUL, IRA, KLM, NW, RAM, SAU, SYR, TWA, TUN. & .03 & .06 & .03 & .23 & \\
\hline & 5 & AC, ANZ, ANA, ANS, BMI, EMI, LFH, MEX, SAS, SIN, SAA, TAI, UA, VIR. & .06 & .06 & .05 & .02 & .38 \\
\hline \multirow{5}{*}{1999} & 1 & LIN, ALG, AOM, CYP, EL, FIN, OLY, RAM, SAB, TAP, TUN. & .24 & & & & \\
\hline & 2 & AFL, AMX, CHI, AFR, IND, AUS, BAL, CSA, DL, IBR, KOR, LOT, MLV, SWR, TAR, UKR. & .09 & .41 & & & \\
\hline & 3 & AC, ANZ, ANA, ANS, BMI, EMI, LFH, MEX, SAS, SIN, SAA, TAI, UA, VRG, VIR. & .01 & .05 & .42 & & \\
\hline & 4 & ALA, ALI, AW, AA, BA, CAI, CO, JAS, JA, KLM, LCH, NW, QUA. & .05 & .10 & .05 & .38 & \\
\hline & 5 & CRO, EGY, GUL, IRA, MA, RAJ, SLA, SYR, TWA, THY. & .03 & .11 & .07 & .04 & .31 \\
\hline \multirow{5}{*}{2000} & 1 & LIN, ARG, AA, BA, CAI, CP, EL, FIN, LCH, QUA, SAB, TAP. & .41 & & & & \\
\hline & 2 & AFL, AFR, ALI, AUS, BAL, CRO, CSA, IBR, IRA, JA, LOT, MLV, RA, SWR, SYR, TAR, THY. & .13 & .43 & & & \\
\hline & 3 & AMX, ALG, CYP, DL, EGY, GUL, OLY, RAM, TUN. & .05 & .09 & .22 & & \\
\hline & 4 & AC, IND, ANZ, ANA, BMI, EMI, LAU, LFH, MA, MEX, SAS, SIN, SAA, SLA, TAI, UA, VIR. & .03 & .09 & .03 & .38 & \\
\hline & 5 & CHI, ALA, AW, ANS, CO, JAS, KLM, KOR, NW, TWA, UKR. & .07 & .06 & .01 & .05 & .29 \\
\hline
\end{tabular}

Fontes: lata's World Air Transport Statistics; Airline Business, diversas edições; análises presentes em Lazzarini (2003).

Notas: a Dados nas diagonais indicam densidade da constelação. Dados que não estão nas diagonais indicam densidade de laço entre membros de constelações e membros de outros grupos. 
aéreo a constelações explícitas e implícitas: $\mathrm{IC}_{\mathrm{ijt}}$, codificado como 1 , no caso de as empresas $i$ e $j$ pertencerem à mesma constelação implícita no ano $t$, e 0 , se ocorrer o contrário; e $\mathrm{EC}_{\mathrm{ij}}$, codificado como 1, no caso de as empresas $i$ e $j$ pertencerem à mesma constelação explícita no ano $t$, e 0 , se ocorrer o contrário.

\section{Variáveis explicativas}

\section{Adesões passadas a constelações}

Foram utilizadas as variáveis $\mathrm{IC}_{\mathrm{ijt}-1}$ e $\mathrm{EC}_{\mathrm{ijt-1}}$ para monitorar as adesões passadas a constelações explícitas e implícitas. Essas variáveis são simplesmente os valores de $\mathrm{IC}_{\mathrm{ijt}}$ e $\mathrm{EC}_{\mathrm{ijt}}$, respectivamente, com um ano de defasagem, sendo utilizadas para o teste das hipóteses $1,2 \mathrm{a}$ e $2 \mathrm{~b}$.

\section{Diversidade de recursos}

Para testar a hipótese 3, foram criadas duas variáveis para medir a diversidade das empresas de transporte aéreo em uma constelação. Primeiro, definiu- se o posicionamento internacional de uma empresa como a razão entre o tráfego (RPK - revenue passenger kilometers) originado dos fluxos internacionais de passageiros em relação ao tráfego total. Em seguida, criouse a variável Divint ${ }_{\mathrm{ij}, \mathrm{t}}$ que é a razão entre a maior e a menor pontuação no posicionamento internacional das empresas $i$ e $j$ no ano $t$. A idéia embutida na utilização dessa variável é a de que a diversidade de recursos em constelações de aviação pode depender do fato de alguns membros se especializarem em mercados pequenos e domésticos, enquanto outros se especializam em grandes mercados, com uma ampla gama de rotas internacionais. Assim, Clougherty (2002) demonstra que grandes redes domésticas podem melhorar o posicionamento competitivo de uma empresa de transporte aéreo em mercados internacionais.

Foi ainda utilizada a variável Dist ${ }_{i j}$, que representa a distância, em quilômetros, entre os principais hubs das empresas i e j. O principal hub de uma empresa de transporte aéreo é definido como a cidade que, para a em-

Tabela 2 - Descrição das constelações explícitas.

\begin{tabular}{|c|c|c|c|}
\hline ANO & NOME & DATA DE FUNDAÇÃO & MEMBROS \\
\hline 1995 & Global Excellence & 1990 & DL, SIN, SWR. \\
\hline 1996 & Global Excellence & 1990 & DL, SIN, SWR. \\
\hline \multirow{3}{*}{1997} & Atlantic Excellence & Fev 1997 & AUS, DL, SAB, SWR. \\
\hline & Global Excellence $^{1}$ & 1990 & DL, SIN, SWR. \\
\hline & Star Alliance & Mai 1997 & AC, LFH, SAS, TAI, UA. ${ }^{2}$ \\
\hline \multirow{3}{*}{1998} & Atlantic Excellence & Fev 1997 & AUS, DL, SAB, SWR. \\
\hline & Qualiflyer & Mai 1998 & AOM, AUS, CRS, LAU, SAB, SWR, TAP, THY. \\
\hline & Star Alliance & Mai 1997 & AC, LFH, SAS, TAI, UA, VRG. \\
\hline \multirow{5}{*}{1999} & Atlantic Excellence $^{3}$ & Fev 1997 & AUS, DL, SAB, SWR. \\
\hline & Oneworld & Set 1998 & $\mathrm{AA}, \mathrm{BA}, \mathrm{CAI}, \mathrm{CP}, \mathrm{QUA}{ }^{4}$ \\
\hline & Qualiflyer & Mai 1998 & AOM, AUS, CRS, SAB, SWR, TAP, THY. ${ }^{5}$ \\
\hline & Star Alliance & Mai 1997 & AC, ANZ, ANS, LFH, SAS, TAI, UA, VRG. \\
\hline & “Wings"6 & 1999 & KLM, NW. \\
\hline \multirow{5}{*}{2000} & Oneworld & Set 1998 & LIN, AA, BA, CP, FIN, IBR, LCH, QUA. \\
\hline & Qualiflyer & Mai 1998 & LIB, AOM, CRS, LOT, SAB, SWR, TAP, THY. ${ }^{7}$ \\
\hline & SkyTeam & Set 1999 & AMX, AFR, DL. ${ }^{8}$ \\
\hline & Star Alliance & Mai 1997 & AC, ANZ, ANA, ANS, AUS, LFH, MEX, SAS, SIN, TAI, UA, VRG. ${ }^{9}$ \\
\hline & “Wings" & 1999 & KLM, NW. \\
\hline
\end{tabular}

Fontes: lata’s World Air Transport Statistics; Airline Business, diversas edições; análises presentes em Lazzarini (2003).

Notas: ${ }^{1}$ Dissolvida em novembro de $1997 ;{ }^{2}$ Varig juntou-se ao grupo em outubro de $1997 ;{ }^{3}$ Dissolvida em novembro de $1999 ;{ }^{4}$ Finnair e lberia juntaram-se ao grupo em setembro de $1999 ;{ }^{5}$ Air Europe também é membro, mas não foi incluída na análise por falta de dados. No entanto, estimativas indicam que ela contribui apenas com aproximadamente $6,2 \%$ do tráfego total da constelação. “ "Wings" é o nome não oficial do grupo. A aliança entre KLM e Northwest existe desde 1989, mas consideramos que o grupo estava oficialmente institucionalizado com o anúncio de que a Continental e a Alitalia se juntariam ao grupo no início de 1999,0 que depois foi cancelado; ${ }^{7}$ Air Littoral, Portugalia e Volare também são membros, mas não foram incluídos na análise por falta de dados. No entanto, estimativas indicam que elas, conjuntamente, contribuem somente para $2,4 \%$ do tráfego total da constelação; ${ }^{8}$ Korean Airlines juntou-se ao grupo em julho de $2000 ;{ }^{9}$ British Midland (bmi) juntou-se ao grupo em julho de 2000. 
presa em questão, mostrar o maior número de conexões de saída, o que é evidenciado pela base de dados Traffic by Flight Stage, compilada pela Organização Internacional de Aviação Civil (OIAC). A utilização dessa variável é justificada com base na premissa de que a diversidade em relação a rotas alternativas oferecidas aos clientes tenderá a ser maior quando os membros estiverem posicionados em cidades distantes, situação que expande as possibilidades de conexões. De modo contrário, hubs similares ou próximos serão mais provavelmente substitutos do que complementares.

Para acomodar possíveis efeitos não-lineares, foi utilizado, nas análises de regressão, o logaritmo das variáveis de diversidade de recursos.

\section{Variáveis de controle}

Laços bilaterais passados

Associações bilaterais existentes entre empresas de transporte aéreo provavelmente influenciam as dinâmicas de formação de constelações explícitas e implícitas. Por exemplo, os membros de um grupo explícito podem tentar atrair novas empresas com as quais mantêm laços bilaterais (Gomes-Casseres, 1996, p. 66). Por isso, criou-se a variável Dirt ${ }_{\mathrm{ijt}-1}$, codificada como 1 se as empresas $i$ e $j$ estivessem associadas por meio de alianças bilaterais ou relação de propriedade no ano $t-1$, e 0 se ocorresse o contrário. Porém, associações bilaterais indiretas também merecem ser consideradas (Gulati, 1995b; Gulati e Gargiulo, 1999). Assim, duas empresas não ligadas entre si, mas bilateralmente associadas com o mesmo parceiro, podem se tornar membros da mesma constelação implícita ou explícita. Nesse sentido, foi criada neste estudo a variável Indirt ijt-1 $_{\text {, }}$, codificada como 1 se as empresas $i$ e $j$ estivessem bilateralmente associadas com ao menos um parceiro comum no ano $t-1$, e 0 se ocorresse o contrário.

\section{Número de constelações explícitas}

$\mathrm{Na}$ medida em que as constelações explícitas são formadas, elas podem se tornar progressivamente institucionalizadas como novas e legítimas formas organizacionais no setor (Garcia-Pont e Nohria, 2002; Gulati e Gargiulo, 1999; Hannan e Freeman, 1989). Clientes, fornecedores e as próprias empresas de transporte aéreo podem se tornar progressivamente conscientes dos benefícios derivados do pertencimento a grupos explícitos - programas comuns de milhagem, serviços conjuntos, operações compartilhadas etc. Assim, a formação de constelações explícitas tenderá a ser reforçada pelo número de constelações existentes. Para controlar esse efeito, foi adicionada a variável Ectot ${ }_{\mathrm{t}-1}$, que corresponde ao número de alianças explícitas observadas no ano anterior ao da análise. Nas regressões, foi utilizado o logaritmo dessa variável para se observar possíveis efeitos não-lineares.

\section{Tamanho}

Para controlar as diferenças de tamanho ou capacidade entre as empresas, criou-se a variável $\mathrm{CAP}_{\mathrm{ijt}}$, que representa a razão entre a capacidade de assentos de passageiros disponíveis para ambas as empresas - do valor maior ao menor - no ano t (Gulati e Gargiulo, 1999). Novamente utilizou-se o logaritmo dessa variável na regressão.

\section{Controles específicos do ano}

Finalmente, foi criado um grupo de variáveis dicotômicas representando cada ano na janela de observação, denominado de $\mathrm{ANO}(\mathrm{t})$, para controlar efeitos temporais, como variações em condições econômicas e regulatórias ao longo do tempo, bem como tendências nos padrões de aliança entre as empresas. Uma verificação do coeficiente de correlação das variáveis (veja Tabela 3) não indica problemas aparentes de colinearidade.

\section{METODOLOGIA}

Uma vez que o estudo trabalha com duas variáveis dependentes dicotômicas, codificando as adesões conjuntas nas constelações explícitas e implícitas, seria possível a utilização de modelos de escolha binários separados - por exemplo: probit ou logit - para o teste de hipóteses. No entanto, há um problema ao se testar essas hipóteses separadamente. Trata-se do fato de que as escolhas são, provavelmente, influenciadas por fatores não observados e que, conjuntamente, afetam a decisão da empresa de aderir ao mesmo grupo explícito ou implícito.

Por exemplo, duas empresas de transporte aéreo poderiam criar acordos particulares, não observados neste estudo e que, simultaneamente, as induzissem a fazer parte de uma mesma constelação explícita e a promover a reorganização dos laços bilaterais, de maneira tal que também se tornassem membros do mesmo grupo implícito. Considerando-se essa possibilidade, foi usado aqui o modelo probit bivariado (Greene, 2000), que estima duas regressões com variáveis dicotômicas relacionadas, modelando dessa forma uma possível correlação entre fatores estocásticos 
- não observáveis - que afetam ambas as escolhas. Formalmente, o modelo é especificado como:

$$
\begin{aligned}
& y_{i j t}{ }^{E}=x_{i j t} \beta^{E}+\varepsilon_{i j t}{ }^{E} \\
& y_{i j t}{ }^{1}=x_{i j t} \beta^{1}+\varepsilon_{i j t}{ }^{I}
\end{aligned}
$$

em que $\mathrm{EC}_{\mathrm{ijt}}=1$ (as empresas $i$ e $j$ pertencem ao mesmo grupo explícito no ano t) se $\mathrm{y}_{\mathrm{ijt}}{ }^{\mathrm{E}}>0$, e 0 se ocorrer o contrário; $\mathrm{IC}_{\mathrm{ijt}}=1$ (as empresas $i$ e $j$ pertencem ao mesmo grupo explícito) se $\mathrm{y}_{\mathrm{ijt}}{ }^{\mathrm{I}} \neq>0$, e 0 se ocorrer o contrário; $x_{\mathrm{ijt}}$ é um vetor de covariâncias; $\beta^{\mathrm{E}}$ e $\beta^{1}$ são vetores parâmetros a serem estimados; e $\varepsilon_{\mathrm{ijt}}{ }^{\mathrm{E}}$ e $\varepsilon_{\mathrm{ijt}}{ }^{\mathrm{I}}$ são termos estocásticos conjuntamente distribuídos de acordo com uma normal bivariada com correlação $\rho$.

Tendo em vista que as escolhas de parcerias são observadas por diversos anos, foi possível calcular desvios-padrão robustos agrupados em cada par de empresas de transporte aéreo.

\section{RESULTADOS E DISCUSSÃO}

A Tabela 4 apresenta estimativas das regressões do modelo probit bivariado. O Modelo 1 apresenta os resultados somente com as variáveis de controle, e o Modelo 2 adiciona o grupo de variáveis explicativas para o teste de hipóteses. Um teste Wald indica que as variáveis explicativas melhoram significativamente $\mathrm{o}$ ajuste da regressão $-\chi^{2}=1279.99, \mathrm{p}<0.01$. Em todos os casos, a estimativa de $\rho$ é significativamente diferente de zero, sugerindo que o modelo probit bivariado é apropriado, visto que fatores não observados, envol- vidos na decisão de escolha de uma empresa de transporte aéreo por grupos explícitos e implícitos, induzem uma correlação dos erros das regressões $-\mathrm{p}<0.01$. Um outro modo de avaliar é fazer a comparação dos resultados do modelo probit bivariado com os resultados obtidos da aplicação de dois modelos probit independentes. Novamente, um teste Wald comparando o ajuste relativo dessas especificações alternativas favorece o modelo probit bivariado $-\chi^{2}=19.06 \mathrm{e}$ 27.82 para os Modelos 1 e 2 , respectivamente, ambos para $\mathrm{p}<0.01$.

Cabem alguns comentários sobre as variáveis de controle, que apresentaram resultados interessantes. Os coeficientes significativamente positivos de Dirt $\mathrm{ijt}_{\mathrm{j}-1}$ e Indirt ${ }_{\mathrm{ijt}-1}$ indicam que, na presente amostra, as empresas que mantinham laços bilaterais umas com as outras, ou associações indiretas por meio de um parceiro comum, apresentaram maior probabilidade de se tornarem membros de um mesmo grupo explícito ou implícito $-\mathrm{p}<0.01$. Isso sustenta a idéia de que as empresas tendem a formar associações de múltiplas partes baseadas em agentes com os quais já estão vinculadas de forma bilateral.

A variável Ln $\left(\right.$ Ectot $\left._{\mathrm{t}-1}\right)$, por sua vez, fornece diferentes sinais, dependendo de a constelação ser implícita ou explícita. Observou-se que um aumento no número de constelações explícitas formadas no ano anterior aumenta, significativamente, a probabilidade de que duas empresas de transporte aéreo se tornem membros do mesmo grupo explícito $-\mathrm{p}<0.01$. A proliferação de grupos explícitos, no entanto, reduz a probabilidade de que duas empresas de transporte aéreo

\begin{tabular}{|c|c|c|c|c|c|c|c|c|c|c|c|c|c|}
\hline \multirow{2}{*}{\multicolumn{2}{|c|}{ VARIÁVEIS }} & \multirow{3}{*}{$\begin{array}{l}\text { MÉDIA } \\
0.016\end{array}$} & \multirow{3}{*}{$\begin{array}{c}\text { DESVIO } \\
\text { PADRÃO } \\
0.124\end{array}$} & \multicolumn{10}{|c|}{ CORRELAÇÕES } \\
\hline & & & & \multirow{2}{*}{1} & \multirow[t]{2}{*}{2} & \multirow[t]{2}{*}{3} & \multirow[t]{2}{*}{4} & \multirow[t]{2}{*}{5} & \multirow[t]{2}{*}{6} & \multirow[t]{2}{*}{7} & \multirow[t]{2}{*}{8} & \multirow[t]{2}{*}{9} & \multirow[t]{2}{*}{10} \\
\hline 1 & $E C_{i j t}$ & & & & & & & & & & & & \\
\hline 2 & $I_{i j t}$ & 0.141 & 0.348 & 0.126 & 1 & & & & & & & & \\
\hline 3 & $E C_{i j t-1}$ & 0.008 & 0.091 & 0.597 & 0.080 & 1 & & & & & & & \\
\hline 4 & $I C_{i j t-1}$ & 0.138 & 0.345 & 0.119 & 0.418 & 0.101 & 1 & & & & & & \\
\hline 5 & $\operatorname{Ln}_{\left(\text {Divint }_{\mathrm{ijt}}\right.}$ ) & 0.675 & 0.843 & -0.010 & -0.090 & -0.012 & -0.075 & 1 & & & & & \\
\hline 6 & $\operatorname{Ln}\left(\right.$ Dist $\left._{\mathrm{ij}}\right)$ & 8.512 & 1.165 & -0.028 & -0.085 & -0.034 & -0.084 & 0.168 & 1 & & & & \\
\hline 7 & Dirt $_{\mathrm{jt}-1}$ & 0.080 & 0.271 & 0.183 & 0.307 & 0.170 & 0.346 & -0.071 & -0.148 & 1 & & & \\
\hline 8 & Indirtt $_{\mathrm{jt}-1}$ & 0.383 & 0.486 & 0.125 & 0.260 & 0.107 & 0.288 & -0.179 & -0.063 & 0.177 & 1 & & \\
\hline 9 & $\operatorname{Ln}\left(\right.$ Ectot $\left._{\mathrm{t}-1}\right)$ & 0.944 & 0.697 & 0.095 & -0.009 & 0.073 & -0.007 & 0.000 & 0.000 & 0.024 & 0.081 & 1 & \\
\hline 10 & $\operatorname{Ln}\left(\mathrm{CAP}_{\mathrm{ijt}}\right)$ & 1.500 & 1.090 & -0.047 & -0.052 & -0.037 & -0.059 & -0.004 & 0.024 & -0.015 & -0.065 & -0.003 & 1 \\
\hline
\end{tabular}

\section{Tabela 3 - Estatística descritiva $(\mathrm{N}=16.650)$.}


se tornem membros do mesmo grupo implícito no futuro $-\mathrm{p}<0.01$. Esse é um resultado de difícil explicação. Talvez a variável Ln (Ectot ${ }_{\mathrm{t}-1}$ ) esteja simplesmente capturando alguma tendência de momento na evolução de constelações implícitas. A Tabela 1 mostra que, nos últimos períodos, não há concentração de empresas em um único grupo implícito.

O coeficiente da variável de controle final, Ln $\left(\mathrm{CAP}_{\mathrm{ijt}}\right)$, revela que empresas com maior diferença em tamanho - capacidade - têm uma probabilidade significativamente maior de se aliarem por meio de uma constelação explícita ou implícita. Dessa forma, empresas com capacidades igualmente grandes podem se ver muito mais como competidoras do que como possíveis parceiras, ao passo que empresas com capacidades igualmente pequenas podem não ter escala suficiente para garantir participação em constelações cujas operações possuam alcance global.

Vejamos agora os resultados das variáveis explicativas introduzidas no Modelo 2. A hipótese 1, no modelo aqui apresentado, pode ser testada pela comparação dos coeficientes de $\mathrm{EC}_{\mathrm{ijt}-1}$ e $\mathrm{IC}_{\mathrm{ijt}-1}$, que representam a participação passada em constelações explícitas e implícitas, respectivamente. Visto que o coeficiente de $\mathrm{EC}_{\mathrm{ijt}-1}$ é positivamente significativo na re- gressão para constelações explícitas, e o coeficiente de $\mathrm{IC}_{\mathrm{ijt}-1}$ é positivamente significativo na regressão para constelações implícitas $-\mathrm{p}<0.01-$, há evidência de inércia, ou seja, as empresas tendem a permanecer na mesma constelação ao longo do tempo. Além disso, o coeficiente $\mathrm{EC}_{\mathrm{ijt}-1}$ na regressão para constelações explícitas é significativamente maior em magnitude do que o coeficiente de $\mathrm{IC}_{\mathrm{ijt}-1}$ na regressão para constelações implícitas $-\chi^{2}=131.02, \mathrm{p}<0.01$. Isso sustenta a hipótese 1: a inércia tende a ser mais forte em constelações explícitas do que nas implícitas, provavelmente porque as constelações explícitas envolvem investimentos irrecuperáveis e compromissos contratuais abrangentes.

As hipóteses $2 \mathrm{a}$ e $2 \mathrm{~b}$ revelam previsões sobre a coevolução de constelações explícitas e implícitas. Para a sustentação da hipótese $2 \mathrm{a}$, o coeficiente de $\mathrm{IC}_{\mathrm{ijt}-1}$ deve ser positivo na regressão das constelações explícitas. E foi exatamente isso o que se observou neste estudo: a participação em uma constelação implícita no passado leva a uma participação futura na mesma constelação explícita $-\mathrm{p}<0.01$. A hipótese $2 \mathrm{~b}$, por sua vez, prevê que o coeficiente de $\mathrm{EC}_{\mathrm{ijt-1}}$ deve ser positivo na regressão para constelações implícitas. Essa hipótese foi rejeitada pelos resultados aqui obtidos: a

Tabela 4 - Estimativas do modelo probit bivariado ${ }^{\text {a }}$.

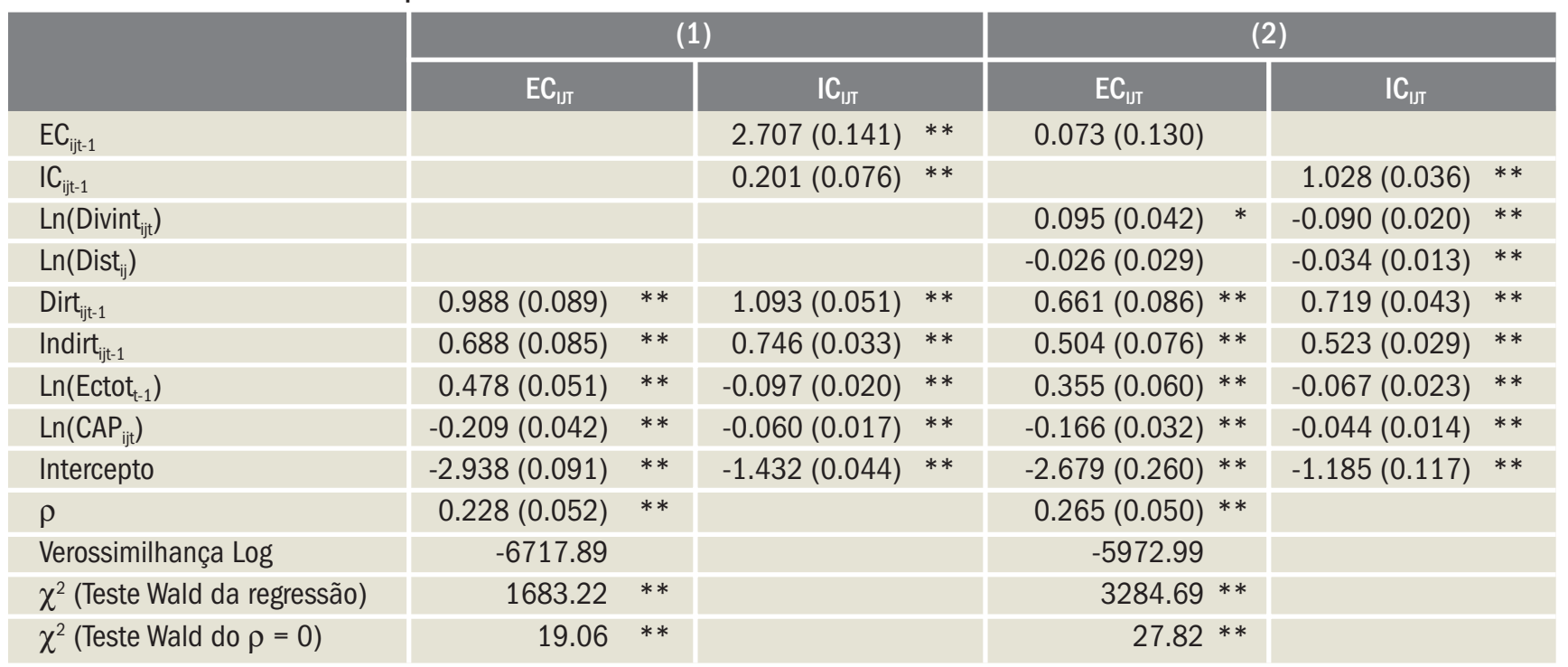

Notas: a $\mathrm{N}=16.429$. Estimativas robustas de erro padrão em parênteses, agrupando-se em cada par de empresas

ij. Todos os modelos incluem variáveis dicotômicas específicas ao ano (não relatadas aqui).

$* * p<0.01$

$* p<0.05$ 
participação na mesma constelação explícita no passado não leva, significativamente, a uma participação futura na mesma constelação implícita.

Esses resultados confirmam que constelações implícitas podem ser "precursoras" de grupos explícitos, mas rejeitam a idéia de que a participação na mesma constelação explícita tende a promover a reorganização dos laços bilaterais de forma que as empresas se tornem membros do mesmo grupo implícito. Uma possível explicação para isso pode ser o fato de que os membros de grupos explícitos tendem a focar em áreas de interesse estratégico coincidentes - por exemplo, rotas transatlânticas e suas extensões continentais -, deixando outras atividades a cargo dos laços bilaterais que são independentemente administrados pelas empresas, de acordo com suas estratégias locais. Em outras palavras, a formação de um grupo explícito não impede parcerias com outros grupos de empresas por meio de constelações implícitas.

No entanto, os membros de uma constelação implícita provavelmente se tornarão, no futuro, membros da constelação explícita à qual seus principais parceiros pertencem. É importante destacar que esse resultado foi observado mesmo depois de controlar os laços bilaterais passados, diretos e indiretos, entre as empresas. Assim, as empresas de transporte aéreo que apresentam maior probabilidade de se tornarem membros de uma mesma constelação explícita não são somente aquelas com laços bilaterais diretos ou indiretos com membros da constelação explícita, mas aquelas associadas ao mesmo grupo de empresas, possuindo mais laços umas com as outras do que com outras empresas na indústria - isto é, empresas de transporte aéreo que pertencem à mesma constelação implícita.

Finalmente, a hipótese 3 prevê que a formação de uma constelação explícita deveria ser mais fortemente influenciada pela diversidade de recursos dos parceiros do que pela formação de constelações implícitas. É possível testar essa hipótese avaliando o coeficiente das duas mensurações deste estudo para diversidade de recursos, a saber: a diferença no posicionamento internacional dos parceiros - Ln (Divint $\left.\mathrm{ijt}_{\mathrm{t}}\right)$ - e a distância entre os principais hubs das empresas - Ln ( Dist $_{\mathrm{ijt}}$ ).

O coeficiente de Ln ( Divint $_{\mathrm{ijt}}$ ) apresentou-se significativamente positivo na regressão das constelações explícitas $-\mathrm{p}<0.05-$, mas significativamente negativo na regressão para constelações implícitas. Tal resultado sustenta a hipótese 3 de maneira ainda mais forte do que era previsto: a diversidade de recursos não só influencia a formação de constelações explícitas, como também está negativamente associada à formação de constelações implícitas. Quanto à Ln (Dist $\mathrm{ijt}_{\mathrm{j}}$, , o coeficiente dessa variável mostrou-se insignificante na regressão para constelações explícitas, mas negativamente significante na regressão para grupos implícitos $-\mathrm{p}<0.01$. Dessa forma, a hipótese 3 é rejeitada quando se considera a distância entre os principais hubs dos parceiros como medida de diversidade de recursos. No entanto, esse resultado confirma que a similaridade de recursos possui papel importante na formação de constelações implícitas. Aparentemente, isso reflete as escolhas envolvidas na exploração da diversidade de recursos. Ao mesmo tempo em que a diversidade aumenta as externalidades que os membros da constelação podem potencialmente internalizar, essa internalização é particularmente difícil de ocorrer no caso de a aliança não possuir acordos formais e entidades coordenadoras, que estão disponíveis nas constelações explícitas, mas ausentes nas implícitas. Assim, enquanto parceiros com recursos diversos possuem maior probabilidade de se tornarem membros de constelações explícitas, parceiros com recursos semelhantes possuem maior probabilidade de se tornarem membros de um mesmo grupo implícito.

Tal resultado sugere uma dinâmica interessante para a co-evolução de grupos implícitos e explícitos. Constelações explícitas podem se expandir pela incorporação de empresas que apresentem recursos diversos e que sejam mais bilateralmente associadas a membros-chave do grupo explícito do que a outras empresas no setor. Ao mesmo tempo, membros de um grupo explícito podem criar associações implícitas com parceiros que possuam recursos mais similares aos seus, criando assim um agrupamento de empresas adjacente à constelação explícita. Uma vez constituído, o grupo explícito exibirá inércia: os membros tenderão a permanecer juntos por algum tempo, pelo menos um tempo maior do que as associações implícitas entre empresas.

\section{COMENTÁRIOS FINAIS}

Este estudo contribui para a literatura sobre formação de alianças entre empresas ao ir além do foco em laços bilaterais para avaliar como as alianças entre múltiplas empresas, comumente chamadas de constelações, evoluem ao longo do tempo.

Cumpre frisar, porém, que este é um estudo exploratório, com limitações importantes. O setor de avia- 
ção apresenta diversas particularidades que impedem a generalização dos resultados a outros contextos. Por exemplo, o tráfego internacional no setor é fortemente regulamentado, o que certamente influencia as decisões de alianças. O setor também testemunhou uma expansão nos laços bilaterais e nas constelações que pode não ter correspondência em outros contextos.

No entanto, este estudo pode servir como guia para pesquisas futuras em outros setores. O setor de microprocessadores é um bom exemplo. As empresas não só estabeleceram agrupamentos de alianças bilaterais - envolvendo, por exemplo, marketing e licenciamento de tecnologia -, mas também consórcios formais para Pesquisa \& Desenvolvimento e produção (Axelrod et al., 1995; Hwang e Burgers, 1997; Vanhaverbeke e Noorderhaven, 2001). Da mesma maneira que o presente estudo, futuras pesquisas poderiam analisar como essas associações formais e informais entre empresas se transformam ao longo do tempo e tentar encontrar medidas de diversidade de recursos para testar as hipóteses aqui sugeridas.

É importante ainda destacar que, embora a composição de grupos explícitos seja facilmente observável por meio de fontes públicas, a análise de constelações implícitas depende crucialmente de métodos quantitativos utilizados para separar as empresas em grupos. Apesar de o algoritmo de otimização tabu search representar uma melhoria com relação a outros métodos usuais, como a análise hierárquica de cluster, ele ainda requer suposições restritivas sobre os laços entre empresas - por exemplo, qual o número "ideal" de divisões a serem encontradas?

Um modo possível de refinar o método é considerar a definição operacional de uma constelação implícita como um grupo de empresas que possua uma quantidade maior de laços entre si do que com empresas de fora do grupo. Observemos que, ao aumentar o número de divisões, é provável que a densidade dos laços intra-grupos também aumente, porém às custas de um aumento na proporção de laços fora do grupo (Bock e Husain, 1950). Uma função "ajuste" que capture essa escolha pode ser incorporada ao algoritmo e guiar a otimização da composição dos grupos e do número de divisões empregado.

Finalmente, a janela temporal utilizada nesta pesquisa pode não ser longa o suficiente para capturar completamente a dinâmica das constelações. Mesmo no setor de aviação, constelações implícitas e explícitas são constantemente formadas e dissolvidas. A observação da evolução entre grupos durante um perío- do maior de tempo pode, possivelmente, revelar outros padrões dinâmicos que não puderam ser aqui avaliados - por exemplo, possíveis ciclos na formação dos grupos. Esse esforço crítico de coleta e análise de dados é deixado para futuros trabalhos.

\section{REFERÊNCIAS BIBLIOGRÁFICAS}

AXELROD, R.; MITCHELL, W.; THOMAS, R. E.; BENNETT, D. S.; BRUDERER, E. Coalition formation in standard-setting alliances. Management Science, v. 41, n. 9, p. 1493-1508, 1995.

BAKER, C. The global groupings. Airline Business, p. 40-45, 2001.

BLAU, P. Exchange and power in social life. New York: Wiley, 1964.

BOCK, R. D.; HUSAIN, S. Z. An adaptation of Holzinger's B-Coefficients for the analysis of sociometric data. Sociometry, v. 13, n. 2, p. 146-153, 1950 .

BORGATTI, S. P.; EVERETT, M.; FREEMAN, L. C. Ucinet 5.0. Natick: Analytic Technologies, 1999.

BURGERS, W. P.; HILL, C. W. L.; KIM, W. C. A theory of global strategic alliances: the case of the global auto industry. Strategic Management Journal, v. 14, n. 6, p. 419-432, 1993.

CAVES, R. E.; PORTER, M. E. From entry barriers to mobility barriers: conjectural decisions and contrived deterrence to new competition. The Quarterly Journal of Economics, v. 91, n. 2, p. 241-261, 1977.

CHUNG, S.; SINGH, H. Complementarity, status similarity and social capital as drivers of alliance formation. Strategic Management Journal, v. 21, n. 1, p. 1-23, 2000.

CLOUGHERTY, J. A. US domestic airline mergers: the neglected international determinants. International Journal of Industrial Organization, v. 20, n. 4, p. 557-576, 2002.

DARR, E. D.; KURTZBERG, T. R. An investigation of partner similarity dimensions on knowledge transfer. Organizational Behavior and Human Decision Processes, v. 82, n. 1, p. 28-44, 2000.

DAS, T. K.; TENG, B. S. Alliance constellations: a social exchange perspective. Academy of Management Review, v. 27, n. 3, p. 445-456, 2002.

DOMOWITZ, I. Electronic derivatives exchanges: implicit mergers, network externalities, and standardization. The Quarterly Review of Economics and Finance, v. 35, n. 2, p. 163-175, 1995.

FARRELL, J.; SALONER, G. Coordination through committees and markets. Rand Journal of Economics, v. 19, n. 2, p. 235-252, 1988. 
GARCIA-PONT, C.; NOHRIA, N. Local versus global mimetism: the dynamics of alliance formation in the automobile industry. Strategic Management Journal, v. 23, n. 4, p. 307-321, 2002.

GLOVER, F. Tabu search - part I. Orsa Journal on Computing, v. 1, n. 3, p. 190-206, 1989.

GNYAWALI, D. R.; MADHAVAN, R. Cooperative networks and competitive dynamics: a structural embeddedness perspective. Academy of Management Review, v. 26, n. 3, p. 431-445, 2001.

GOMES-CASSERES, B. Group versus group: how alliance networks compete. Harvard Business Review, v. 72, n. 4, p. 62-74, 1994.

GOMES-CASSERES, B. The alliance revolution: the new shape of business rivalry. Cambridge: Harvard University Press, 1996.

GRANDORI, A.; SODA, G. Inter-firm networks: antecedents, mechanisms and forms. Organization Studies, v. 16, n. 2, p. 183-214, 1995.

GREENE, W. H. Econometric analysis. Upper Saddle River: Prentice-Hall, 2000.

GULATI, R. Does familiarity breed trust? The implications of repeated ties for contractual choice in alliances. Academy of Management Journal, v. 38, n. 1, p. 85-112, 1995a.

GULATI, R. Social structure and alliance formation pattern: a longitudinal analysis. Administrative Science Quarterly, v. 40, n. 4, p. 619-652, $1995 b$

GULATI, R. Alliances and networks. Strategic Management Journal, v. 19, n. 4, p. 293-317, 1998.

GULATI, R.; GARGIULO, M. Where do interorganizational networks come from? American Journal of Sociology, v. 104, n. 5, p. 1.439-1493, 1999.

HANLON, P. Global airlines: competition in a transnational industry. 2. ed. Oxford: Butterworth Heinemann, 1999.

HANNAN, M. T.; FREEMAN, J. Organizational ecology. Cambridge: Harvard University Press, 1989.

HUMAN, S. E.; PROVAN, K. G. Legitimacy building in the evolution of small-firm multilateral networks: a comparative study of success and demise. Administrative Science Quarterly, v. 45, n. 2, p. 327-365, 2000.

HWANG, P.; BURGERS, W. P. The many faces of multi-firm alliances: lessons for managers. California Management Review, v. 39, n. 3, p. 101$117,1997$.
JONES, C.; HESTERLY, W. S.; FLADMOE-LINDQUIST, K.; BORGATTI, S. P. Professional service constellations: how strategies and capabilities influence collaborative stability and change. Organization Science, v. 9 , n. 1, p. 396-410, 1998.

JOSHI, M. P.; KASHLAK, R. J.; SHERMAN, H. D. How alliances are reshaping telecommunications. Long Range Planning, v. 31, n. 4, p. 542548, 1998.

KATZ, M. L.; SHAPIRO, C. Systems competition and network effects. Journal of Economic Perspectives, v. 8, n. 2, p. 93-115, 1994.

KRAATZ, M. S. Learning by association? Interorganizational networks and adaptation to environmental change. Academy of Management Journal, v. 41, n. 6, p. 621-643, 1998.

LAWLESS, M. W.; ANDERSON, P. C. Generational technological change: effects of innovation and local rivalry on performance. Academy of Management Journal, v. 39, n. 5, p. 1185-1217, 1996.

LAZZARINI, S. G. The performance implications of membership in competing firm constellations: evidence from the global airline industry. Academy of Management Best Paper Proceedings, 2003.

LEVINTHAL, D. A.; FICHMAN, M. Dynamics of interorganizational attachments: auditor-client relationships. Administrative Science Quarterly, v. 33, n. 3, p. 345-369, 1988.

LEVINTHAL, D. A.; MARCH, J. G. The myopia of learning. Strategic Management Journal, v. 14, n. 8, p. 95-112, 1993.

LI, S. X.; ROWLEY, T. J. Inertia and evaluation mechanisms in interorganizational partner selection: syndicate formation among U.S. investment banks. Academy of Management Journal, v. 45, n. 6, p. 11041119,2002

MACNEIL, I. R. Values in contract: internal and external. Northwestern University Law Review, v. 78, n. 2, p. 340-418, 1980.

MADDALA, G. S. Limited-dependent and qualitative variables in econometrics. Cambridge: Cambridge University Press, 1983.

MARTIN, X.; PARK, N. K. Why firms enter into successive international alliances: bounded momentum and alliance formation. New York: Stern School of Business, 2002. (Working paper).

MCPHERSON, J. M. Hypernetwork sampling: duality and differentiation among voluntary organizations. Social Networks, v. 3, n. 4, p. 225-249, 1982.

NOHRIA, N.; GARCIA-PONT, C. Global strategic alliances and industry structure. Strategic Management Journal, v. 12, n. 4, p. 105-124, 1991. 
OUM, T. H.; YU, C. Winning airlines: productivity and cost competitiveness of the world's major airlines. Boston: Kluwer Academic Publishers, 1998.

PARK, J. H.; ZHANG, A. An empirical analysis of global airline alliances: cases in North Atlantic markets. Review of Industrial Organization, v. 16, n. 4, p. 367-383, 2000 .

PARK, N. K.; MARTIN, X. When do resources enhance firm value? Testing alliance and resource effects on firm value. New York: Stern School of Business, 2001. (Working paper).

RICHARDSON, G. B. The organization of industry. The Economic Journal, v. 82, n. 327 , p. 883-896, 1972.

RING, P. S.; VAN DE VEN, A. H. Developmental processes of cooperative interorganizational relationships. Academy of Management Review, v. 19, n. 1, p. 90-118, 1994.

SCHILLING, M. A.; STEENSMA, H. K. The use of modular organizational forms: an industry-level analysis. Academy of Management Journal, v. 44, n. 6, p. 1149-1168, 2001.

Texto traduzido por Rebeca Alves Chu. Artigo recebido em 09.06.2003. Aprovado em 06.01.2004.
STUART, T. E. Network positions and propensities to collaborate: an investigation of strategic alliance formation in a high-technology industry. Administrative Science Quarterly, v. 43, p. 668-698, 1998.

TEECE, D. J. Competition, cooperation, and innovation: organizational arrangements for regimes of rapid technological progress. Journal of Economic Behavior \& Organization, v. 18, n. 1, p. 1-25, 1992.

TER KUILE, A. Hub fever. Airline Business, v. 11, n. 12, p. 66-71, 1997.

THOMPSON, J. D. Organizations in action: social science bases of administrative theory. New York: McGraw-Hill, 1967.

VANHAVERBEKE, W. NOORDERHAVEN, N. G. Competition between alliance blocks: the case of the Risc microprocessor technology. Organization Studies, v. 22, n. 1, p. 1-30, 2001.

WALKER, G. Network analysis for cooperative interfirm relationships. In: CONTRACTOR, F. J.; LORANGE, P. (Eds.). Cooperative strategies in international business: joint ventures and technology partnerships between firms. Lexington: Lexington Books, 1988. p. 227-240.

\section{Sérgio G. Lazzarini}

Professor das Faculdades Ibmec-SP. Doutor em Administração pela John M. Olin School of Business, Washington University. Interesses de pesquisa em alianças e redes de empresas, co-opetição, e dinâmicas competitivas.

E-mail: sergiogl1@ibmec.br

Endereço: Rua Maestro Cardim, 1170 - São Paulo - SP, 01323-001.

\section{Thiago A. Z. Joaquim}

Graduado em Administração de Empresas pelas Faculdades Ibmec-SP. Interesses de pesquisa em dinâmicas competitivas e estratégia empresarial.

E-mail: thiagoazj@ibmec.br

Endereço: Rua dos Franceses, 174, 61-B - São Paulo - SP, 01329-010. 